\title{
46. SOME REMARKS ON THE LIBERATION OF GASES FROM COMETARY NUCLEI
}

\author{
B. YU. LEVIN \\ O. Schmidt Institute of Physics of the Earth, Moscow, U.S.S.R.
}

\begin{abstract}
According to modern data water ice is the main constituent of cometary nuclei. The rate of its evaporation determines the rate of liberation of other constituents, including those that determine the photometric properties of comets. The estimates of the total mass loss per perihelion passage seem to give values about one order of magnitude smaller than those necessary to explain the nongravitational forces as due to the reactive force of material leaving the nuclei. Thus the conventional explanation of the nongravitational forces deserves further study from the point of view of the physical theory of comets.
\end{abstract}

\section{Introduction}

About 20 years ago Dubyago (1948) and Whipple (1950) ascribed the acceleration and deceleration of motion of some periodic comets (Encke, Biela, Brooks 2, and others) to the reactive force of material leaving the surface of a rotating cometary nucleus. Since then this explanation of the nature of nongravitational forces influencing the motions of some comets - both periodic and nonperiodic - has become widely accepted.

However, one difficulty exists: to explain the values of the nongravitational forces derived from observations, a mass loss of a few percent per perihelion passage is necessary (Dubyago, 1948; Whipple, 1950; Sekanina, 1969). Smaller values published by several authors are underestimates because the numerical value of the directional factor used in the calculations was 3 to 5 times too large. According to modern ideas on the evaporation of icy cometary nuclei, a mass loss of the order of 0.1 to $1 \%$ per perihelion passage is possible, but it seems impossible to explain larger mass losses.

Let us discuss this difficulty in detail.

\section{Evaporation of Water Ice and the Photometric Properties of Comets}

The discovery of the presence of atomic oxygen in cometary heads is important evidence which leads, with other evidence, to the conclusion that water ice (or snow) is the major constituent of cometary nuclei. For water ice, the heat of evaporation is large $\left(L=12000 \mathrm{cal} \mathrm{mol}^{-1}=670 \mathrm{cal} \mathrm{g}^{-1}\right)$ and, as was shown by several authors (Biermann and Trefftz, 1964; Huebner, 1965; Delsemme, 1966; Rijves, 1966, 1970), its sublimation from an exposed nucleus unprotected by a nonvolatile insulating layer explains the production rate of $\mathrm{H}_{2} \mathrm{O}$ molecules derived from the observed intensities of the $\mathrm{O}$ and $\mathrm{OH}$ lines. Smaller values of $L$ derived earlier from the rate of variation of the so-called integral brightness with heliocentric distance, and based on the hypothesis that gases are desorbed from the nonvolatile layer, are fictitious for two reasons:

(1) For $L=12000 \mathrm{cal} \mathrm{mol}^{-1}$ the zone where comets are usually observed ( $\left.r \approx 1 \mathrm{AU}\right)$ 
corresponds to transition from intense sublimation (evaporation) at small heliocentric distances $r$ to slow sublimation at large $r$. At sufficiently small $r$ almost all the solar heat absorbed by the nucleus is used for sublimation of ice, and here the direct production of gas molecules from the nucleus as well as the liberation of nonvolatile dust particles varies according to the $1 / r^{2}$ law. According to the experiments by Delsemme and Wenger (1970), grains of water ice must be stripped from the surface of the nucleus by the outstreaming gas. These grains evaporate when moving through the coma and thus increase the total gas production.. It is probable that the ejection of grains also follows the $1 / r^{2}$ law, and consequently the total gas production $Q$ follows this law at small $r$.

On the other hand, when the comet is far from the Sun almost all the solar heat is reemitted in the form of thermal radiation, and here $Q$ closely follows the $\exp (-L / R T)$ law, where the effective temperature $T$ is determined by radiative equilibrium $(T=$ $\left.T_{0} \sqrt{ } r\right)$.

In the transition zone, both sublimation and reradiation play an important role in the heat balance (Delsemme, 1966). In this zone inappropriate application of the desorption formula leads to fictitious numerical values of $L$ much smaller than 12000 cal mol-1.

(2) The analysis of photometric observations of comets by means of the desorption formula was based on the proportionality between $Q$ and the true integral brightness of the head I (Levin, 1943, 1947, 1948). However, observations usually give, not the total integral brightness $I$, but only the integral brightness $I^{\prime}$ of the central part of the head. If the limiting isophote has a constant brightness $B$, then $I^{\prime} \sim Q^{2} / r^{4}$. But for many comets observed at $r<1 \mathrm{AU}$ the decrease in $r$ (and increase in $Q$ ) is accompanied by a decrease in the elongation of the comet from the Sun and, consequently, by an increase in the brightness of the sky background. Therefore, $B$ increases, and this alters the dependence of $I^{\prime}$ on $Q$ and $r$. For most comets it is thus impossible to derive the correct dependence of $Q$ on $r$ from the observed 'integral' brightness of the head.

Formerly (Levin, 1966), I regarded the strong dependence of integral brightness on heliocentric distance $r$ - much stronger than the $1 / r^{2}$ law - as an argument in favour of desorption of gases from a nonvolatile layer and against the sublimation of exposed ices. This was because at that time I thought the low values of $L$ obtained from the photometric observations were real. If one assumes that cometary nuclei are composed of volatile ices with $L \approx 3000-5000 \mathrm{cal} \mathrm{mol}^{-1}$ and unprotected by a nonvolatile layer, then at heliocentric distances of the order of $1 \mathrm{AU}$ the sublimation would be intense and would follow the $1 / r^{2}$ law. But this is not the case. At the present time the photometric behaviour of bright comets can be explained as due to the decisive role of the less volatile $\mathrm{H}_{2} \mathrm{O}$ ice in the composition of nuclei. The rate of sublimation of the water ice determines the rate of liberation of all the other components, both more volatile and less.* For faint comets the observed integral brightness deviates substantially

\footnotetext{
* As some components of cometary nuclei are more volatile than water ice, and as nonvolatile components and grains of water ice are ejected without evaporation (Huebner and Weigert, 1966; Delsemme and Wenger, 1970; Delsemme and Miller, 1970), the effective heat of evaporation (per gram of ejected material) is somewhat smaller than $670 \mathrm{cal} \mathrm{g}^{-1}$.
} 
from the true brightness, and this has an additional influence on their photometric behaviour.

The smaller brightness of periodic comet Encke after perihelion, which I regarded as further evidence in favour of the desorption hypothesis (Levin, 1943, 1966), must now be explained in some other way, perhaps as a systematic error due to difference in observing conditions (brightness of sky background, etc.).*

Thus I withdraw my previous objections against the evaporation of exposed ices and agree that the total rate of gas production in comets is determined mainly by the rate of evaporation of the water ice. Therefore, the so-called Levin formula cannot be used to compute the latent heat of gas production in comets.

\section{Evaporation of Exposed Ices and the Necessary Mass Loss}

In the case of evaporation of exposed ices, although many details remain unclear or even unknown, it is possible to evaluate the thickness of the surface layer lost during one perihelion passage, as was calculated by Huebner (1967). For a typical comet with its perihelion inside the orbit of the Earth the thickness of this layer is a few metres, and it reaches a few tens of metres for sungrazing comets (Huebner, 1967). Since the relative mass loss is given by

$$
\frac{\Delta M}{M}=\frac{4 \pi R^{2} \Delta R}{\frac{4}{3} \pi R^{3}}=\frac{3 \Delta R}{R},
$$

we obtain, for a nucleus with $R=3 \mathrm{~km}$ and $\Delta R=3 \mathrm{~m}, \Delta M / M \sim 0.3 \%$.

For smaller nuclei the mass loss can reach $1 \%$, while for larger nuclei it is of the order of $0.1 \%$. These numerical values for the mass loss are in the same range as those calculated by Dubyago (1948), Whipple (1950), and Sekanina (1969) to explain the nongravitational forces. However, all these authors used in their calculations a value of about 0.5 for the directional factor. This value seems somewhat high even for the radial component of the reactive force produced by evaporation from the sunlit hemisphere of a nonrotating nucleus. But it is several times too high when applied to the transverse component connected with the rotation of the nucleus. For a directional factor of about 0.1 Sekanina (1969) obtained for three comets mass loss rates of 0.1 to $0.5 \%$, but for three others he obtained rates of $1 \%$ or more. The case of $\mathrm{P} / \mathrm{Sch}$ wassmann-Wachmann 2 is especially strange because of its large perihelion distance $q(2.1 \mathrm{AU})$, while $\Delta R$ should decrease with increasing $q .^{\dagger}$

Thus there is a discrepancy of one order of magnitude between the value of the mass loss necessary to explain the nongravitational forces and that accounted for by the modern physical theory of comets.

* For nonperiodic comets (1915 II Mellish, 1965 VIII Ikeya-Seki) the similar effect can be explained by the development of a radial gradient in the composition of the outermost layers of the nucleus. + In the zone where the $1 / r^{2}$ law is applicable $\Delta R$ decreases proportionally to $q^{-1 / 2}$, if the role of eccentricity is neglected (Huebner, 1967), while at larger $r$ it decreases more rapidly (Levin, 1947, p. 208). 


\section{Position of Maximum Ejection of Material}

As cometary nuclei are composed of snow with low thermal conductivity, the surface temperature of a nucleus must quickly adjust to changes in the incident solar radiation caused by the nuclear rotation. At small $r$ this is achieved by the use of solar heat for evaporation of ice, while at larger $r$ both evaporation and reradiation play an important role. The maximum surface temperature must be close to the subsolar point.

Observations of comets seem to confirm these expectations. Drawings by visual observers of central parts of the coma, as well as photographs on which these parts are not overexposed, show emission fans directed towards the Sun. This can be seen on several figures in the Atlas of Cometary Forms by J. Rahe, B. Donn, and K. Wurm, on

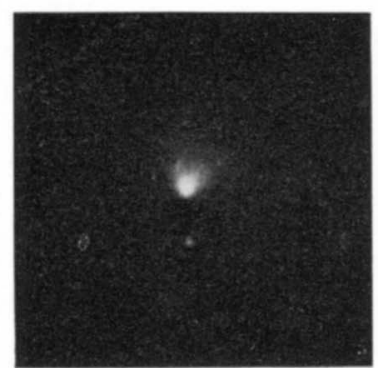

Fig. 1. Comet $1932 \mathrm{~V}$ (Peltier-Whipple) on 1932 August 24-25.

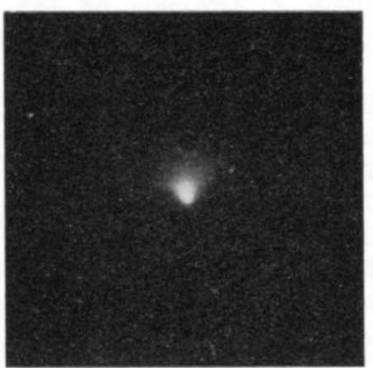

Fig. 2. Comet 1932 V (Peltier-Whipple) on 1932 August 26-27.

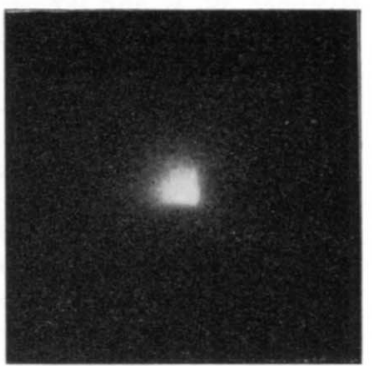

Fig. 3. Comet 1936 II (Peltier) on 1936 July 26-27.

some photographs of P/Encke (Roemer, 1961), and on unpublished photographs of comets 1932 V (Peltier-Whipple) and 1936 II (Peltier) taken for astrometric purposes with the 38-cm double astrograph at the Moscow Observatory (see Figures 1-3).

Although studies of the precise directions of these fans are lacking, it seems obvious that there are no large deviations from the sunward direction.

In most cases the behaviour of the nongravitational forces is in line with these data: the radial component is usually directed away from the Sun and is an order of magnitude larger than the transverse component (Marsden, 1969). Negative values of the radial component were recently found by Marsden (1970) for periodic comets Encke, d'Arrest, Wirtanen, Arend, and perhaps Pons-Winnecke, but the precision of these negative values is poor.

In all studies of nongravitational forces the possible displacement of the photometric centre of the asymmetric image of a comet from the true position of the nucleus was not taken into account. The probable importance of this effect was stressed many years ago by Mokhnach (1956), and the observations of the comet 1957 III (ArendRoland) seem to confirm it (Debehogne, 1968). This effect must be taken into account in the study of such a delicate problem as nongravitational forces in comets. 


\section{Conclusion}

At the present time there are difficulties concerning the amount of the mass loss from cometary nuclei. Resolution of them is indispensable for explaining the presence of nongravitational forces.

\section{References}

Biermann, L. and Trefftz, E.: 1964, Z. Astrophys. 59, 1.

Debehogne, H.: 1968, Acad. Roy. Belg. Bull. Cl. Sci. 54, 1040.

Delsemme, A. H.: 1966, Mem. Soc. Roy. Sci. Liège Ser. 5 12, 69, 77.

Delsemme, A. H. and Miller, D. C.: 1970, Planetary Space Sci. 18, 717.

Delsemme, A. H. and Wenger, A.: 1970, Planetary Space Sci. 18, 709.

Dubyago, A. D.: 1948, Astron. Zh. 25, 361.

Huebner, W. F.: 1965, Z. Astrophys. 63, 22.

Huebner, W. F.: 1967, Z. Astrophys. 65, 185.

Huebner, W. F. and Weigert, A.: 1966, Z. Astrophys. 64, 185.

Levin, B. Yu.: 1943, Dokl. Akad. Nauk SSSR 38, 82; Astron. Zh. 20 (4), 37.

Levin, B. Yu.: 1947, Usp. Astron. Nauk 3, 191.

Levin, B. Yu.: 1948, Astron. Zh. 25, 246.

Levin, B. Yu.: 1966, Mem. Soc. Roy. Sci. Liège Ser. 5 12, 65.

Marsden, B. G.: 1969, Astron. J. 74, 720.

Marsden, B. G.: 1970, Astron. J. 75, 75.

Mokhnach, D. O.: 1956, Byull. Inst. Teor. Astron. 6, 269.

Rijves, V. G. : 1966, Komety $i$ Meteory No. 3, 3.

Rijves, V. G.: 1970, Komety $i$ Meteory No. 17, 3.

Roemer, E.: 1961, Publ. Astron. Soc. Pacific 73, 170.

Sekanina, Z.: 1969, Astron. J. 74, 1223.

Whipple, F. L.: 1950, Astrophys. J. 111, 375.

\section{Discussion}

A. Z. Dolginov: What is the basis for your statement that the nucleus consists mainly of water ice?

B. $Y u$. Levin: Estimates of the amount of gas evaporated from the nucleus, based on the hypothesis of water ice, are in agreement with observed intensities of atomic oxygen and hydroxyl in the head.

$H$. Alfvén: If the evaporation velocity is of the order of at most $100 \mathrm{~m} \mathrm{~s}^{-1}$, the maximum value of $\Delta V$ necessary to account for the nongravitational effects cannot very much higher. Is this in agreement with the observed values of the nongravitational effects?

B. Yu. Levin: I think that we need an order of magnitude increase, either in the mass of matter ejected or in the velocity. The calculation allows for the relative velocity of the material leaving the nucleus, assumed to be $500 \mathrm{~m} \mathrm{~s}^{-1}$.

D. O. Mokhnach: I want to say some words in support of nongravitational forces, and to refute my views of 15 years ago. Vsekhsvyatskij has shown that the secular decrease in the brightness of $\mathrm{P} /$ Encke is 2 to 2.5 magnitudes per 50 revolutions. Even if we assume that the decrease is only one magnitude, then the decrease in the evaporating surface of the nucleus would be as much as $60 \%$ and the corresponding mass loss would be about $25 \%$. Most of the mass loss takes place near perihelion, and in fact, the mass loss in other parts of the orbit may be neglected. Hence, it was reasonable for Makover to assume that an abrupt acceleration occurs at the perihelion point. There are other effects associated with the variations in total brightness, such as the deformation of isophotes, and the deflection of the photometric centre; and systematic discrepancies between the mass centre and photometric centre can result only in an increase of the residuals of the orbit calculation. However, the mass loss, which is probably greater than I have calculated, yields nongravitational effects that may easily result in acceleration or deceleration of the comet's motion. 